\title{
Laboreal
}

Volume $9 \mathrm{~N}^{\circ} 2$ | 2013

Varia

\section{¿Puede hablarse de transferencia de modelos de investigación/herramientas de intervención en ergonomía?}

Podemos falar em transferência de modelos de investigação/ferramentas de intervenção em ergonomia?

Peut-on parler de transfert de modèles de recherche / outils d'interventions en ergonomie?

Can we talk about transfer of research models / tools interventions in ergonomics?

\section{Abada Mhamdi}

\section{OpenEdition}

\section{Journals}

Edición electrónica

URL: http://journals.openedition.org/laboreal/5612

DOI: 10.4000/laboreal.5612

ISSN: 1646-5237

Editor

Universidade do Porto

Referencia electrónica

Abada Mhamdi, « ¿Puede hablarse de transferencia de modelos de investigación/herramientas de intervención en ergonomía? », Laboreal [En línea], Volume 9 №2 | 2013, Publicado el 01 diciembre 2013, consultado el 04 octubre 2019. URL : http://journals.openedition.org/laboreal/5612 ; DOI : $10.4000 /$ laboreal. 5612

Este documento fue generado automáticamente el 4 octubre 2019.

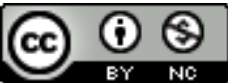

Laboreal está licenciado com uma Licença Creative Commons - Atribuição-NãoComercial 4.0 Internacional. 


\section{¿Puede hablarse de transferencia de modelos de investigación/ herramientas de intervención en ergonomía?}

Podemos falar em transferência de modelos de investigação/ferramentas de intervenção em ergonomia?

Peut-on parler de transfert de modèles de recherche / outils d'interventions en ergonomie?

Can we talk about transfer of research models / tools interventions in ergonomics?

Abada Mhamdi

\section{NOTA DEL EDITOR}

Manuscrito recibido en : Octubre/2013

Aceptado tras peritaje : Noviembre/2013

\section{Introducción}

1 Diversas experiencias en las empresas tunecinas muestran que la herramienta o el método de investigación ergonómico se construyen in loco. Cada intervención es un caso particular y las diversas contribuciones de la ergonomía o la clínica de la actividad ya han proporcionado los fundamentos teóricos y las explicaciones pertinentes. De esta forma, se sabe que es raro que un método desarrollado en una empresa pueda trasladarse (aplicarse) tal cual a otro lugar. No obstante, la problemática adopta otra configuración cuando la "transferencia" o la apropiación se realizan en el contexto de 
un país con "ergonomía emergente". A través de un ejemplo concreto, vamos a realizar un primer balance de las cuestiones en juego. Primero presentaremos una metodología de investigación/acción que pusimos en marcha en Francia y que quisimos retomar posteriormente en Túnez. Luego, presentaremos nuestras problemáticas con respecto a la transferencia y la apropiación de modelos teóricos metodológicos, analizando en qué medida y cómo pueden tenerse en cuenta los entornos particulares de trabajo.

\section{Fundamentos teóricos}

2 El trabajo es un objeto de conocimientos orientados hacia la acción. Partiendo de este postulado, la reflexión sobre el trabajo y por el trabajo favorece, por consiguiente, la adquisición de conocimientos ya que el propio objeto de la reflexión es el trabajo (Schön, 1994 ; Argyris \& Schön, 1989 ; Argyris, 1995 ; Teiger, 1993 ; Teiger \& Lacomblez, 2005 ; 2006). Esta reflexión sobre el trabajo puede ser individual. Es una reflexión sobre la acción y en la acción. Puede ser igualmente colectiva, como en la presente investigación, que se basa en los principios fundamentales del paradigma de la formación de los agentes para y por la acción.

Debe precisarse que el objeto de nuestras preocupaciones es replantear la prevención de los accidentes de trabajo y contribuir a reducir su frecuencia y gravedad. De esta forma, compartimos con otras personas la idea de una "prevención general" es decir, el principio de un enfoque que recuse la exterioridad y el carácter normativo de una prevención prescriptiva. En efecto, diferentes trabajos, en concreto los procedentes de la ergonomía, han puesto de manifiesto la fertilidad del lugar de trabajo en materia de aprendizaje y cómo el "trabajo de concienciación de las herramientas cognitivas puestas en marcha de manera implícita en las actividades prácticas permiten su movilización en la confrontación de situaciones desconocidas" (Malglaive citado in Clot, 1995, p. 38).

4 Aquí se atribuye un lugar esencial a lo que Cru y Dejours (1983) denominaron el "saber hacer de prudencia". Dicho de otra forma, se parte de la base de que los operadores conocen, más o menos implícitamente los peligros de su trabajo, y que se defienden concretamente con ayuda de procedimientos específicos articulados en el curso del propio trabajo, desarrollados a estos efectos, compartidos o individuales. Este "saber hacer de prudencia" está constituido, por consiguiente, por conocimientos prácticos (parcialmente) inconscientes, construidos en la dinámica del colectivo de trabajo e indisociables del saber hacer profesional.

$5 \quad$ En términos metodológicos, los trabajos de Teiger y Laville (1991) han servido de base. En efecto, a partir de los años 70, y como consecuencia de las demandas sindicales, los autores pusieron a punto su método de análisis de trabajo guiado e interactivo -un método que ha sido ampliamente utilizado desde entonces, en particular en la formación de agentes de prevención.

6 En el plano teórico, se parte de la presuposición de una "formación para y por la acción" que podrá situarse en el marco de una "ciencia de la acción" (Teiger, 1993; Teiger \& Montreuil, 1995, Teiger \& Lacomblez, 2013). Dicho de otra forma, la construcción (gracias al autoanálisis del trabajo y a la actividad del colectivo que lo acompaña en sesión) de nuevas representaciones del trabajo, más ricas y cercanas de la complejidad de la actividad real, abre otros campos conceptuales y otras perspectivas 
de intervenciones (Lacomblez, Montreuil \& Teiger, 2000), que permanecieron hasta entonces en la sombra del respeto de las normas de producción.

7 Partimos, por consiguiente, del principio de que existe, previamente a una eventual acción de formación, una apropiación de las técnicas -y eventualmente una adaptaciónpor parte de los protagonistas del trabajo, un terreno propicio y que favorece la puesta en marcha de estas acciones. Pero también sabemos que esta apropiación se realiza en general en el marco, por una parte, de fuertes exigencias de tiempo y, por otra, de relaciones jerárquicas que limitan los "campos de lo posible" de este saber hacer en materia de prudencia, y obligan a los trabajadores a adaptarse al trabajo tal y como es. Por lo tanto, sostenemos que el tiempo dedicado a una acción de formación o reflexión sobre el trabajo y por el trabajo resulta potencialmente beneficioso para los operadores que participan en la misma, pero no a cualquier precio.

En este sentido, y continuando con un enfoque crítico de las acciones de formación denominadas tradicionales (puntuales, descontextualizadas y "castradoras" de la experiencia ya adquirida por los participantes), suscribimos, al igual que otros, esta reflexión de Sartre (1943), que lleva a privilegiar el nivel de la transformación de las representaciones, ya que nos parece efectivamente esencial en este proceso que precede a la acción. No obstante, este proceso es sin duda más complejo de lo que parece : no se trata de un simple "detonante" de una toma de conciencia, y lo que nos interesa realmente es la complejidad de lo que puede ocurrir.

Dando prioridad de esta forma a momentos de actividades reflexivas y discursivas (en el trabajo, sobre el trabajo y para el trabajo), hemos tratado, por lo tanto, de debatir las representaciones de nuestros interlocutores con relación a su trabajo y a las condiciones en que este se realiza. Hemos intentado enfocar los problemas de salud y seguridad en el trabajo desde un enfoque holístico, integrado y centrado en la finalidad de la acción.

\section{El contexto de la estrategia inicial}

El pedido de la empresa de electricidad francesa Electricité de France (EDF) se basaba en una preocupación específica sobre los accidentes de trabajo de origen eléctrico : solo representan un $4 \%$ del conjunto de los accidentes de trabajo, pero suelen ser muy graves.

11 La formulación del pedido aspiraba a entender mejor los accidentes de origen eléctrico $\mathrm{y}$, más concretamente, a investigar los factores que determinan los comportamientos de los operadores con relación a la seguridad, en particular, el uso de los equipos de protección personal (EPP) y el seguimiento de las instrucciones. Efectivamente, la mayoría de los análisis posteriores a los accidentes, realizados por las empresas (informes de síntesis de los accidentes y análisis) destacan principalmente el "factor humano" ; que puede adoptar diversas expresiones : no utilizar los EPP en el momento de los accidentes, incumplir las buenas prácticas y las instrucciones de seguridad, etc.

Pero el análisis de los accidentes de trabajo no basta para desarrollar o reforzar el control de los riesgos en el trabajo. Por lo tanto, es necesario desarrollar otros enfoques. Algunos recurren a grabaciones de vídeo y actividades en situación real de trabajo con tres características principales :

- las actividades en cuestión son realizadas por los propios operadores ; 
- la finalidad es prevenir accidentes ;

- la aspiración es la elaboración de referentes comunes como prerrequisitos para la emergencia o el desarrollo de prácticas de un saber hacer en materia de seguridad.

Durante el análisis de la actividad laboral de los operadores de EDF-GDF SERVICES (en la actualidad denominada: Electricité Réseau Distribution France: ERDF), constatamos que en las agencias [1] en las que se practican talleres-escuelas no se producen accidentes de origen eléctrico (Mhamdi, 1996). El principio de estos talleres-escuela consiste en filmar en situación real, con una cámara de vídeo, una operación delicada realizada o un nuevo método de trabajo con el personal de la agencia. Efectivamente, el control de proximidad y la dirección decidieron, en colaboración con los sindicatos, poner en marcha un proyecto de actualización de conocimientos teóricos y prácticos a través del uso del vídeo como herramienta de formación. No se cuestionan en ningún caso los conocimientos de los agentes ni tampoco se los sanciona tras el análisis del caso estudiado en el taller-escuela. Se proyecta después la película, seguida de un debate crítico sobre la intervención por los propios agentes, en presencia de sus supervisores. Esto tiene una doble finalidad: (i) enriquecer los conocimientos y las prácticas de campo y (ii) partir de las insuficiencias observadas para mejorar la prevención y el saber hacer profesional.

14 La participación en el taller-escuela no se impone a los operadores, que aprenden el método al aceptar proponer situaciones de trabajo para filmar. La razón esencial de esta elección está motivada por el profundo convencimiento de que una toma de conciencia eficaz y positiva de los operadores en materia de seguridad supone su implicación voluntaria.

El objetivo es contribuir a la construcción de nuevos conocimientos orientados directamente a la práctica en el terreno. Se hace una puesta en común de la manera de actuar con seguridad. Esta actividad reflexiva en el trabajo orientaría las nuevas formas de actuar y de trabajar. La construcción de conjuntos de conocimientos se orienta a la práctica y a la transformación de las representaciones.

Nuestra hipótesis era que existía un vínculo posible entre la práctica de los talleresescuela y la ausencia de estos accidentes. Por ello, sin pretender explicar este vínculo de manera general, hemos buscado pistas de explicación analizando lo que ocurría en estos talleres-escuela en un afán por entenderlo mejor.

Para responder a este objetivo, hemos analizado el contenido de las reuniones de debate colectivo en las que se proyecta el film de vídeo del taller-escuela. Hemos dado a estos debates colectivos la siguiente denominación : "ARCAV" (acrónimo de Actividades de Reflexión Colectiva Asistida por Vídeo). Desde el punto de vista metodológico, las ARCAV se definen como "sesiones de reflexión colectiva, deliberación y debate con proyección de películas de taller-escuela cuyo análisis prolongan". Durante estas ARCAV, los operadores pueden explicitar sus actividades de trabajo. Esta explicitación constituye la oportunidad, a través de su propio ejercicio, de una concientización y una puesta en común de experiencias individuales sobre el trabajo. Se trata igualmente de una acción de actualización de los conocimientos adquiridos en la acción por la movilización de diferentes representaciones. Uno de los resultados esperados de este enfoque es que estas reuniones desarrollen prácticas más seguras por parte de los agentes (Mhamdi, 1998a). 
18 El interés práctico de este método estribaría en poner de relieve la variedad de puntos de vista, significaciones y lógicas emergentes. La confrontación de estas últimas tiene como finalidad la puesta en común de experiencias y la construcción de referentes comunes o la "construcción de entornos cognitivos compartidos" (Sperber y Wilson, 1986-1989).

\section{El modo operatorio de las ARCAV}

19 Para entender la lógica subyacente de estos talleres-escuela, preguntamos a los supervisores y agentes si podían dedicar más tiempo al debate que versó sobre la actividad, métodos, medios puestos en marcha, herramientas empleadas, etc. (Mhamdi, 1998a).

20 El principio de las ARCAV consistía en filmar al grupo de agentes que veían la película del taller-escuela realizado con anterioridad, tras pedirles su autorización para filmar las reuniones durante las cuales el grupo debate acerca de las situaciones filmadas. Asisten a las ARCAV los agentes (montadores de línea, capataces y agentes de "pequeñas intervenciones") y sus superiores jerárquicos cercanos (supervisores, jefes de agencia, jefes de agencia adjuntos, y un experto en materia de prevención y seguridad: el ingeniero de seguridad). El número varía en cada ARCAV : entre 7 y 23 operadores (Mhamdi, 1998b). Sus edades oscilan entre los 30 y los 54 años, con una antigüedad de entre 6 y 20 años. El ergónomo graba en vídeo las ARCAV y solo interviene para solicitar aclaraciones.

21 En estas ARCAV, la reflexión se integra en el tiempo de trabajo ya que entra en el marco de un plan de acción prevención que permite a los agentes reunirse para reflexionar sobre la mejora de las prácticas en el trabajo. Las ARCAV duran entre 3 y 4 horas que se reparten en : 1 hora y media para ver el vídeo del taller escuela y entre 1 hora y media y 2 horas para el debate y la reflexión colectiva sobre el film presentado. Podemos decir que su producción es de orden cognitivo : construcción y elaboración de conocimientos y de un saber hacer, transformación de las representaciones de los participantes, adquisición de prácticas meta funcionales, etc.

Destacamos que los operadores de base, confrontados a situaciones de trabajo reales, heredan de hecho la totalidad de los disfuncionamientos e imprevistos que pueden presentarse en el proceso. In vivo, elaboran prácticas de trabajo, en general individuales, a veces colectivas, que valen la pena dar a conocer al colectivo. El objetivo de esta investigación consiste en parte en suscitar las reacciones y debates de los operadores : esto constituye una fase previa indispensable para la puesta en marcha de acciones concretas, ya que, desde nuestro punto de vista, las actividades de reflexión y debate sobre el trabajo y por el trabajo permiten elaborar nuevas "normas" en materia de seguridad del grupo por parte de este mismo. De esta forma, las ARCAV consisten en hacer que los operadores se expresen acerca de sus prácticas y representaciones frente a una situación determinada, y a actuar de forma que se apropien colectivamente de la "mejor" práctica, ya se trate de una práctica individual o de la integración de varias de ellas :

- apropiarse por sí mismo de las prácticas de otros cuando estas resultan eficaces individualmente,

- identificar las demás prácticas individuales para integrarlas en su propio funcionamiento dentro del equipo. 
Esto permite asimismo que los más jóvenes se apropien de la experiencia de los más veteranos y favorece la creación de una cultura del debate, el diagnóstico y la identificación de situaciones de riesgo, el análisis y la propuesta de soluciones comunes, realizables y que gocen del acuerdo de todos los participantes, incluyendo a los superiores.

24 La información sacada de la proyección de vídeo de las películas de los talleres-escuela sirve como situación representativa para el debate en lo relativo a la prevención, la formación y el perfeccionamiento de conocimientos. En esto estriba la originalidad del método y su aportación se sitúa en los siguientes niveles :

- el análisis general de los debates,

- la formalización e interpretación (mirada del investigador),

- la trazabilidad del contenido de la reunión,

- la transmisión posible de estos resultados a otros lugares.

Añadimos que EDF se ha apropiado las ARCAV adaptándolas. Contrató a un cineasta profesional, quien produjo 4 vídeos sobre las condiciones reales de trabajo. Dichos vídeos se integraron en la formación de todos los agentes de EDF para usarse como talleres-escuela.

\section{La puesta en marcha de las ARCAV en Túnez}

Hemos querido poner en marcha las ARCAV en Túnez en el medio hospitalario tunecino (Mhamdi, Ladhari, Hamzaoui, Ben Salah \& Gharbi, 2009). La intervención se realizó en la unidad de diálisis de un hospital de la región del gran Túnez. El médico responsable de esta unidad de diálisis solicitó la realización de un estudio ergonómico sobre las condiciones de trabajo. El pedido estaba justificado por las quejas de "dolor de espalda" del personal. En medio hospitalario, las actividades siguen siendo esencialmente manuales : la falta de espacio o la falta de personal obligan a los operadores a realizar actividades de manipulación pesada en posturas forzadas e incorrectas. En este ejemplo, la unidad de diálisis está instalada en una sala con una superficie de $170 \mathrm{~m}^{2}$. Incluye 20 camas, 20 máquinas (entre las camas) y 20 mesas. El personal oscila entre 20 y 25 trabajadores, entre ellos 3 auxiliares de clínica, 4 obreros y 1 vigilantes general. Para cada sesión de diálisis están presentes en la sala de diálisis 5 o 6 enfermeras, 3 obreros y 2 mujeres de la limpieza. La manipulación de las camas y sus ajustes se realiza mediante manivelas incorporadas en las camas. La unidad funciona los 7 días de la semana, día y noche.

La edad promedio del personal era de 40 años, siendo 28 años la edad del más joven y 58 años la del más mayor. Tenían una antigüedad media en la unidad de diálisis de 10 años. Un $60 \%$ eran mujeres. Las dos sesiones de ARCAV se realizaron con dos grupos : uno compuesto por 6 enfermeros y el otro por 10. Los superiores dieron su consentimiento para que se hiciera el estudio pero sin participar en el mismo.

De esta intervención se desprende que, pese a los obstáculos que surgieron en la fase previa al estudio, este se realizó finalmente, pero solo una parte del personal participó. Los no participantes se mostraron muy reticentes alegando su desconfianza en cuanto al logro de los objetivos, poco convencidos de que la dirección pusiera en marcha los medios necesarios (financieros y reglamentarios) para cualquier propuesta de cambio, corrección o compras de nuevos materiales. 

de trabajo eran forzadas y muy duras. Quedó patente que la manipulación de materiales no ajustables (camas, máquinas), así como numerosas situaciones o cargas pesadas debían levantarse o manipularse manualmente (enfermos, recipientes voluminosos, etc.).

Durante estas ARCAV, se propuso equipar a la unidad de diálisis con camas ajustables como las de las unidades de cuidados intensivos, cambiar los asientos y comprar camillas ajustables, revisar la organización del trabajo en la unidad.

31 Además, este estudio pone de manifiesto que los temores de los miembros del personal más reticentes estaban en parte justificados y fue necesario hacer constar que ningún responsable administrativo, susceptible de tomar decisiones, asistió a las ARCAV.

En conclusión, debe admitirse que la dirección no estaba muy implicada y que no asistió a las reuniones con el personal. Las decisiones adoptadas por las ARCAV no fueron seguidas ni realizadas.

\section{Un modelo de intervención relacionado}

Este enfoque se integra en el paradigma de la formación de los agentes para y por la acción que es una referencia esencial (Lacomblez, 2001). En el marco de esta tradición, el objetivo es efectivamente transmitir a los interlocutores la capacidad de analizar las condiciones en las que trabajan : la confrontación de los saberes más teóricos (donde impera una lógica de vínculos de causalidad) y de los saberes más empíricos (donde impera la finalidad de la acción) suscitará una nueva reflexión y respaldará una transformación del trabajo así como otra gestión de los riesgos.

Estos fundamentos teóricos y metodológicos han permitido también a Ricardo Vasconcelos (Vasconcelos \& Lacomblez, 2000 ; Vasconcelos, 2008) elaborar en Portugal su método denominado MAGICA (Método del Análisis Guiado Individual y Colectivo en Alternancia). Hemos querido aprehender la especificidad y evoluciones.

La hipótesis de la intervención, concebida de manera que se facilite la implicación del colectivo y la jerarquía en la construcción de la seguridad, ha involucrado, además de a los operadores, a otros agentes (el personal de dirección directo, los técnicos encargados del mantenimiento, los responsables de la previsión, etc.), teniendo en cuenta otras actividades y no solo las de los "accidentes potenciales", ya que "la seguridad se construye y desconstruye en todo momento, dentro de los límites volátiles de lo posible, en el plano de la intersección de estas actividades con las de otros agentes" (Vasconcelos \& Lacomblez, 2000 ; Vasconcelos, 2008).

El proyecto MAGICA articula además en el marco del proceso de formación momentos de "autoanálisis guiado" de la actividad de los operadores industriales en su puesto de trabajo, y momentos en los que estos datos se comparten y se debaten en un grupo de pares y otros agentes "relativamente pertinentes" dadas las actividades y problemas que se discuten. Habida cuenta de que la finalidad es esbozar soluciones posibles, congruentes con el plan organizativo, y validadas de manera interdisciplinar (recurriendo a ingenieros, médicos del trabajo y responsables en materia de prevención de riesgos laborales).

37 Añadimos que, en los momentos de análisis guiado en el puesto de trabajo, Vasconcelos ha tratado siempre de formalizar y organizar los conocimientos de los trabajadores 
haciendo hincapié en la comprensión de las actividades de trabajo y no en el inventario de las situaciones de riesgo. Los conocimientos iniciales de los operadores se integran entonces en descripciones detalladas de su actividad de trabajo y de los efectos demostrados en el terreno de la salud.

Por otra parte, el proyecto MAGICA apostó por la utilización de procedimientos de mediación, recogiendo, por ejemplo, piezas defectuosas para utilizarlas en una sesión de formación, o definiendo situaciones-problemas (Pastré, 1992, 2000, 2004), partiendo del conocimiento inicial de la actividad laboral en cuestión. El análisis realizado por el equipo de operadores en el proceso de formación y en el de concienciación estuvo respaldado por "mediadores" entre los participantes y su actividad laboral, tanto a través de la mediación social (relativa a la acción del formador y a la de los miembros del equipo), la mediación material (las piezas defectuosas) como la de la medicación simbólica (recurso a situaciones-problemas).

La acción de formación jugó así un papel concluyente en materia de salud y seguridad en el trabajo. A corto plazo, las transformaciones permitieron efectivamente una revisión de algunos aspectos de la situación laboral en cuestión, y diversos indicadores pusieron de manifiesto una reducción significativa del número de accidentes de trabajo (Vasconcelos \& Lacomblez, 2000; Vasconcelos, 2008). Pese a ello, Vasconcelos se cuestiona acerca de la continuidad de los efectos de su intervención.

\section{Una antropotecnología de los modelos de intervención}

Estos ejemplos suscitan cuestionamientos que merecen una reflexión por parte de la comunidad de investigadores y profesionales en el ámbito de la prevención.

Sin duda, puede considerarse que se trata de captar mejor en qué medida y de qué forma los agentes de prevención en primera línea (encargados de seguridad, médicos de trabajo, psicológicos del trabajo, ergónomos, etc.) han aprehendido efectivamente los principios conceptualizados y concebidos por los investigadores.

Pero también llama nuestra atención una segunda cuestión, perteneciente al campo de la antropotecnología (Wisner, 1984): si el modelo de las ARCAV, tal y como fue concebido, fue difícilmente aplicado en la empresa tunecina, ello se debe en gran parte a las particularidades de un contexto en el que las preocupaciones principales estriban en unas condiciones de trabajo extremadamente precarias y, sobre todo, en el mantenimiento del empleo. Pero en este caso, ¿habría podido transmitirse pese a todo (velar por una apropiación) a los agentes de la prevención in loco, la experiencia adquirida en otros lugares?

Partimos de la hipótesis de que el regreso reflexivo a las actividades de investigación anteriores es una "etapa" que puede permitir ilustrar de manera más pertinente las dificultades halladas durante la elaboración de los estudios y su puesta en marcha (Lacomblez, Montreuil \& Teiger, 2000). Pueden destacarse algunos "puntos clave" (De la Garza, 1999) que deben evitarse si nos dotamos de los medios de intentar transmitir el proyecto renovándolo. 

puesta en marcha de las ARCAV en el medio hospitalario tunecino, pueden resumirse en este estadio de nuestra reflexión de la siguiente forma :

- ¿Qué agentes o qué instancias permiten conceder tiempo del tiempo de trabajo para filmar las actividades reales de trabajo, ver la película realizada por el equipo asignado a estos efectos, con objeto de reflexionar colectivamente y debatir para ver qué conclusiones, acciones o recomendaciones pueden obtenerse?

- ¿Cómo emprender este tipo de proyecto en empresas en las que ni siquiera se aplican acciones de prevención "ordinarias" ? Al margen del medio hospitalario, esta cuestión es particularmente delicada para las intervenciones en empresas, independientemente del sector y del país. Si en las empresas tunecinas raramente se ponen en marcha las acciones de prevención más elementales, ¿cómo "aprehender" a través de la transferencia un método como el de las ARCAV, costoso en términos financieros y de tiempo de trabajo ? Este tipo de pregunta se plantea sin duda en otros países en donde la ergonomía es aún "emergente".

- ¿Debe tratarse de evaluar el costo de uso de un método para la empresa y ponderar una eventual "pérdida de ganancias" en la producción en razón de las interrupciones de trabajo ? ¿Podría la empresa negociar en este caso las acciones de formación y acción?

- Es sabido que las "ganancias" de cualquier política de prevención resultan difíciles de cuantificar cuando se trata de accidentes o incidentes evitados. Evidentemente, puede considerarse que la controversia estriba fundamentalmente en el ámbito del debate acerca de los valores, que nada puede justificar que se asuman riesgos en el recurso al trabajo humano. Pero esta "inercia de la prueba" suele conducir de todas formas a privilegiar la mejora del rendimiento visible a corto plazo con objeto de justificar más fácilmente sus acciones de prevención (Amalberti, 1996; Mhamdi, 1998b). La paradoja de las acciones de prevención es que suelen ser costosas desde el punto de vista financiero, y difíciles de justificar desde el punto de vista técnico económico.

A través de cuestiones similares ha evolucionado el método MAGICA, para convertirse en MATRIOSCA (Matriz de Análisis del Trabajo y Riesgos Profesionales destinado a Supervisores, Personal de Dirección y Estructuras de Apoyo), con la finalidad de garantizar el compromiso necesario a largo plazo del equipo de trabajo y de la dirección en la construcción de la seguridad (Vasconcelos, 2008). Ahora bien, en el medio hospitalario tunecino, la dirección no estaba muy implicada y, como hemos destacado, las decisiones adoptadas por las ARCAV no fueron seguidas ni realizadas.

No obstante, compartimos la misma preocupación que Vasconcelos y Lacomblez (2000) acerca de estas actividades de debate por y para el trabajo, tratando de articular, en el marco del proceso de formación, momentos de autoanálisis guiado de la actividad de los operadores, sobre su puesto de trabajo, en los momentos en los que se comparten y debaten estos resultados en un grupo de iguales y otros agentes "relativamente pertinentes" respecto a las actividades y problema debatidos, así como para esbozar posibles soluciones, congruentes en el plano organizativo.

De este proceso de análisis individual y colectivo emerge innegablemente una mejor comprensión de los problemas identificados, intercediendo en la construcción de soluciones, descubriendo igualmente nuevos problemas o implicando a otros agentes que se revelen progresivamente pertinentes para el proceso.

Como indican Vasconcelos y Lacomblez (2000), la investigación, la acción, la formación y la transformación se basan entonces en un proyecto de prevención primaria que pretende ser interactivo y realmente participativo. El psicólogo del trabajo -más que un 
analista del trabajo durante sesiones individuales en el puesto de trabajo, o más que un simple moderador en sesiones de debate y construcción colectiva de soluciones a los problemas identificados- desempeña de esta forma una función primordial de un "custodio de la actividad y de sus interfaces", para retomar la sugerencia de Oddone y Re (1994), es decir: un garante del respeto hacia el trabajo real y la congruencia organizativa de las soluciones esbozadas.

Así, la lógica del proceso de formación supone - como sugiere Maggi (2006) superponer un proceso secundario (la formación) a un proceso primario (la actividad cotidiana de trabajo) que toma el relevo, como un eje principal y primordial en la adquisición del saber hacer y de determinadas habilidades no impartidas en el marco de un plan de estudios y una formación más académica. Ambos avanzan concertadamente, transformándose mutua y progresivamente. La formación acompaña el desarrollo de la actuación de los sujetos, adaptándose a las necesidades que estos manifiestan, tanto en lo relativo a la organización de la formación (contenidos, duración, ritmos, prioridades) como en lo relativo al proceso primario (sus actividades de trabajo), cuyos problemas se identifican, profundizan y, en algunos casos, se resuelven poco a poco, sobre la marcha, gracias a su acción combinada (Vasconcelos, 2008).

\section{Conclusión}

Es este artículo, hemos presentado la historia de una metodología de investigación desarrollada en primer lugar en una empresa francesa. Utilizada por esta, proporcionó talleres-escuela para la formación de sus agentes. No obstante, el mismo método, retomado en Túnez, no ofreció resultados convincentes. Este fracaso se debe en gran parte a la falta de interés de los responsables de la institución y a un contexto diferente: la ausencia de estructuras internas, o suficientemente próximas, permanentes o que se sientan implicadas.

51 Resulta sin duda extraño que un método desarrollado en una empresa pueda transferirse (apropiarse) tal cual a otra. ¿Puede preverse no obstante la transferencia de modelos teórico metodológicos? ¿En qué condiciones puede separarse un método de lo que Yves Schwartz denomina una "extraterritorialidad" (Schwartz, 2012) y tener en cuenta un entorno particular de trabajo, la organización específica de una empresa y la cultura de un país con relación a la percepción de los riesgos, salvaguardando al mismo tiempo orientaciones y principios considerados primordiales? Sin duda, es necesario que estos principios se renueven en un anclaje local. Por esta razón, pensamos que el desarrollo de lugares "locales", creados para facilitar y mantener un "rendimiento reflexivo" -lugares comunes, en interfaz, entre la investigación y la empresa- podrían contribuir a identificar las condiciones necesarias para dotar de una permanencia local- al tratamiento de las cuestiones planteadas, permitiendo así el enriquecimiento de este tipo de debates así como la capitalización de los logros y reveses de las experiencias emprendidas. 


\section{BIBLIOGRAFÍA}

Amalberti, R. (1996). La conduite de systèmes à risques. París : PUF.

Argyris, C. (1995). Savoir pour agir. París: InterEdition.

Argyris, C., \& Schön, D.A. (1989). Organizational learning: a theory of action perspective. Reading (Mass): Addison Wesley.

Clot, Y. (1995). Le travail sans l'homme? Pour une psychologie des milieux de travail et de vie. París : La découverte.

Cru, D., \& Dejours, C. (1983). Les savoir-faire de prudence dans les métiers du bâtiment. Les cahiers médicaux-sociaux, 3, 239-247.

De la Garza, C. (1999). Fiabilité individuelle et organisationnelle dans l'émergence de processus incidentels au cours d'opérations de maintenance. Le Travail Humain, 62(1), 63-91.

Lacomblez, M. (2001). Analyse du travail et élaboration des programmes de formation professionnelle. Relations industrielles /Industrial Relations, 56(3), 543-578.

Lacomblez, M., Montreuil, S., \& Teiger, C. (2000). Ergonomic work analysis, training and action: new paths opened by the interconnection of approaches. In Proceedings of the IEA 2000/HFES 2000 Congress. Santa Monica: Human Factors and Ergonomics Society, pp. 647-650, United States of America.

Maggi, B. (2006). Do agir organizacional. Um ponto de vista sobre o trabalho, o bem-estar, a aprendizagem. São Paulo : Editora Edgard Blucher.

Mhamdi A. (1998b). Les activités de réflexion collective assistées par vidéo : un outil pour la prévention. Thèse d'ergonomie, CNAM, Parí.

Mhamdi A., Ladhari N., Hamzaoui H., Ben Salah, F. et Gharbi, R. (2009). Video assisted training as a tool for prevention: the nurse's case. In Proceeding of the XVIIth IEA Triennial World Congress on Ergonomics, August 9-14, Beijing -China.

Mhamdi, A. (1996). L'analyse statistique de données accidentelles préalable à l'analyse ergonomique du travail. In Actes du $31^{\text {ème }}$ Congrès de la SELF. Bruselas (Bélgica).

Mhamdi, A. (1998a). Activités de Réflexion Collective Assistée par Vidéo : activité productive de nouveaux savoir-faire. In M-F. Dessaigne et I. Gaillard (Eds). Des évolutions en ergonomie (pp. 135-144). Toulouse : Octares Editions.

Oddone, I. \& Re, A. (1994). Come recuperare l'expertise professionale. In E. Tranchina et al. Il Portolano di Psicologi. Pistoia : Centro di documentazione Pistoia.

Pastré, P. (1992). L'analyse du travail en didactique professionnelle, Revue française de pédagogie. 138, 9-17.

Pastré, P. (2000). Signification, sens, formation. París : PUF.

Pastré, P. (2004). Recherches en didactique professionnelle. Toulouse : Octarès.

Sartre, J.P. (1943). L'Être et le néant, essai d'ontologie phénoménologique. París : Gallimard.

Schön, D.A. (1983-1994). Le praticien réflexif : à la recherche du savoir caché dans l'agir professionnel. (J. Heynemand \& D. Gagnon, trad.). Quebec : Les éditions LOGIQUES. 
Schwartz, Y. (2012). Las dos paradojas de Alain Wisner. Antropotecnología y ergología. Laboreal, 8 , (2), 55-73. http://laboreal.up.pt/revista/artigo.php?id=37t45nSU54711246696336752:1 .

Sperber, D., \& Wilson, D. (1986-1989). La pertinence, communication et cognition. París : les éditions de minuit.

Teiger, C. (1993). Représentation du travail, travail de la représentation. In A. Weill-Fassina, P. Rabardel \& D. Dubois (Eds). Représentations pour l'action (pp. 311-344). Toulouse : Octares.

Teiger, C., \& Lacomblez, M. (2005). L'ergonomie et la trans-formation du travail et/ou des personnes (1ère partie). Éducation Permanente, 165, 9-28.

Teiger, C., \& Lacomblez, M. (2006). L'ergonomie et la trans-formation du travail et/ou des personnes (2eme partie). Éducation Permanente, 166, 105-114.

Teiger, C., \& Lacomblez, M. (2013). (Se) former à l'analyse du travail au sein de l'entrepriseatouts et limites de quelques démarches d'intervention. In C. Teiger et M. Lacomblez (Eds). (Se)Former pour transformer le travail. Dynamiques de constructions d'une analyse critique du travail (pp. 369-455). Laval : Presse de l'Université Laval.

Teiger, C., \& Laville, A. (1991). L'apprentissage de l'analyse ergonomique du travail, outil d'une formation pour l'action. Travail et Emploi, 1(47), 53-62.

Teiger, C., \& Montreuil, S. (1995). Les principaux fondements et apports de l'analyse ergonomique du travail en formation. Education permanente, 124, 13-28.

Vasconcelos, R. (2008). o papel do psicólogo do trabalho e a tripolaridade dinâmica dos processos de transformação : contributo para a promoção da segurança e saúde no trabalho. Tese de Doutoramento, Faculdade de Psicologia e Ciências da Educação da Universidade do Porto, Porto.

Vasconcelos, R., \& Lacomblez, M. (2000). Identification and Prevention of Accident Risks through the Development of Self-Analysis-of-Work Competencies among Industrial Workers. In Proceedings of the Human Factors and Ergonomics. Society Annual Meeting, 44(2), 667-670. doi: $10.1177 / 154193120004401260$.

Wisner, A. (1984). L'anthropotechnologie, outil ou leurre? Technologies, Idéologies, Pratiques, 5 , 28-59.

\section{NOTAS}

1. Agencia - organización territorial encargada de:

- el contacto con la clientela ordinaria y la gestión de pequeñas intervenciones y del acceso a las energías para la agencia cliente,

- la gestión del funcionamiento de la red y la construcción de obras de baja tensión para la agencia de explotación. 


\section{RESÚMENES}

Diversas experiencias en las empresas tunecinas muestran que la herramienta o el método de investigación ergonómico se construyen in loco. Cada intervención es un caso particular y las diversas contribuciones de la ergonomía o la clínica de la actividad ya han proporcionado los fundamentos teóricos y las explicaciones pertinentes. Por lo tanto, es raro que un método desarrollado en una empresa pueda trasladarse (aplicarse) tal cual en otro lugar. ¿Puede, no obstante, contemplarse la transferencia de modelos teórico metodológicos ? ¿En qué medida la metodología puede tener en cuenta un entorno particular de trabajo, la organización específica de una empresa y la cultura de un país con relación a la percepción de los riesgos, salvaguardando al mismo tiempo orientaciones y principios considerados primordiales? Construir un lugar común entre la investigación y la empresa deberá permitir conferir una permanencia local a este tipo de debates.

árias experiências nas empresas tunisianas demonstram que a ferramenta ou o método de intervenção ergonómica é construído in loco. Cada intervenção é um caso específico e várias contribuições da ergonomia ou do processo da actividade proporcionaram os seus fundamentos teóricos e explicações. É, portanto, raro que um método desenvolvido numa empresa possa ser transferido (aplicado) conforme o é noutro sítio. A transferência de modelos teóricos e metodológicos pode, no entanto, ser ponderada? Em que medida o processo pode considerar um ambiente específico de trabalho, a organização específica de uma empresa e a cultura de um país relativamente à percepção dos riscos, mantendo em simultâneo orientações e princípios considerados primordiais ? Construir um local comum entre a investigação e a empresa deveria permitir dar uma perenidade local a este tipo de debates.

Plusieurs expériences dans les entreprises tunisiennes montrent que l'outil ou la méthode d'intervention ergonomique se construit in loco. Chaque intervention est un cas particulier et plusieurs contributions de l'ergonomie ou de la clinique de l'activité en ont donné les fondements théoriques et les explications. Il est donc rare qu'une méthode développée dans une entreprise puisse être transférée (appliquée) telle qu'elle l'est ailleurs. Le transfert de modèles théoricométhodologiques peut-il cependant être envisagé ? Dans quelle mesure la démarche peut-elle tenir compte d'un environnement particulier de travail, de l'organisation spécifique d'une entreprise et de la culture d'un pays concernant la perception des risques, tout en sauvegardant des orientations et des principes considérés primordiaux ? Construire un lieu commun entre la recherche et l'entreprise devrait permettre de donner une pérennité locale à ce type de

Several experiences in Tunisian companies show that the tool or methods in ergonomics intervention must be built in loco. Each intervention is a typical case and many contributions of ergonomics or clinical analysis of activity have given the theoretical bases and explanations. It's exceptional that a method developed in a company can be transferred (applied) as it is elsewhere. However, how far can the transfer of theoretical and methodological models be applied? Up to what point can the approach take into account a particular work environment, the specific organization of the company and the culture of a country on risk perception, while safeguarding guidelines and principles considered essential? Building common ground between the company and research is expected to give local sustainability in this type of debate. 
ÍNDICE

Palavras-chave: antropotecnologia, ergonomia, país emergente, modelos de intervenção Palabras claves: antropotecnología, ergonomía, país emergente, modelos de intervención Mots-clés: anthropotechnologie, ergonomie, pays émergent, modèles d'intervention Keywords: anthropotechnology, ergonomics, DCIs, tools, models, intervention, research

\section{AUTOR}

\section{ABADA MHAMDI}

Université Tunis El Manar - Faculté de Médecine de Tunis - Laboratoire de Toxicologie d'ergonomie et d'Enivrement Professionnel, 15, Rue Djebel Lakhdar La Rabta, 1007 Tunis Tunisie

abada.mhamdi@fmt.rnu.tn 